\begin{tabular}{|c|c|}
\hline Title & Blood soluble Fas levels and mortality from cardiovascular disease in middle aged Japanese: The JA CC study \\
\hline Author(s) & I so, Hiroy asu; Maruy ama, Koutatsu; Eshak, Ehab S.; I kehara, Satoyo; Y amagishi, Kazumasa; Tamakoshi, A kiko \\
\hline Citation & $\begin{array}{l}\text { A therosclerosis, 260, 97-101 } \\
\text { https://doi.org/10.1016/.atherosclerosis.2017.03.020 }\end{array}$ \\
\hline Issue Date & 2017-05 \\
\hline Doc URL & http:/hdl.handle.net/2115/70044 \\
\hline Rights & $\begin{array}{l}\text { (9 2017. This manuscript version is made available under the CC-BY -NC-ND } 4.0 \text { license } \\
\text { http://reativecommons.org/icenses/by-nc-nd/4.0/ }\end{array}$ \\
\hline Rights(URL) & http://creativecommons.org/icenses/by-nc-nd/4.0/ \\
\hline Type & article (author version) \\
\hline File Information & A therosclerosis260_97.pdf \\
\hline
\end{tabular}

Instructions for use 


\section{Blood soluble Fas levels and mortality from cardiovascular disease in middle-aged}

\section{Japanese: the JACC Study.}

Hiroyasu Iso, $\mathrm{PhD}^{1^{*}}$; Koutatsu Maruyama, $\mathrm{PhD}^{2}$; Ehab S Eshak, $\mathrm{PhD}^{1,3}$; Satoyo Ikehara, $\mathrm{PhD}^{1,4}$; Kazumasa Yamagishi, $\mathrm{PhD}^{5}$; Akiko Tamakoshi, $\mathrm{PhD}^{6}$

${ }^{1}$ Public Health, Department of Social Medicine Osaka University Graduate School of Medicine, Yamadoka, 2-2 Suita-shi, Osaka, Japan 565-0871.

${ }^{2}$ Department of Public Health, Graduate School of Medicine, Juntendo University, 2-1-1 Hongo, Bunkyo-ku, Tokyo 113-8421, Japan.

${ }^{3}$ Department of Public Health and Preventive Medicine, Faculty of Medicine, Minia University, Main raod, Shalabyland, Minia, Egypt, 61511.

${ }^{4}$ Department of Hygiene and Public Health, Osaka Medical College, 2-7 Daigaku-machi, Takatsuki, Osaka, 569-8686, Japan

${ }^{5}$ Department of Public Health Medicine, Faculty of Medicine, University of Tsukuba, 1-1-1 Tennodai, Tsukuba, Ibaraki, 305-8575,Japan.

${ }^{6}$ Public Health, Department of Social Medicine, Graduate School of Medicine, Kita 15 Nishi 7, Kita-ku, Sapporo, 060-8638 Japan.

*Correspondence:Prof. Hiroyasu Iso

Public Health, Department of Social Medicine, Osaka University, Graduate School of Medicine Suita-shi, Osaka 565-0871 Japan.

TEL: +81-6-6879-3911 FAX: +81-6-6879-3919 E-mail: iso@pbhel.med.osaka-u.ac.jp Cover title: Blood soluble Fas levels and cardiovascular disease.

Number of tables: 2 Figures: 0 Supplemental tables: 1. 
Keywords: biomarkers, stroke; subarachnoid haemorrhage; coronary heart disease; mortality; nested-case control study.

Word count: 2412 


\section{ABSTRACT}

Background and aims: Limited evidence has been available on the relationship between apoptosis with cardiovascular disease in population-based samples. We examined whether blood soluble Fas are associated with mortality from cardiovascular diseases.

Methods: In a nested case-control study under a large prospective cohort; the Japan Collaborative Cohort (JACC) Study, where a total of 39,242 subjects 40 to 79 years of age provided serum samples and followed up for 9 years, we measured blood soluble Fas (sFas) levels among cases and controls, matched for sex, age, area of residence and year of serum storage. Conditional logistic regression models were used to calculate odds ratio (95\%CI) of mortality from stroke and stroke subtypes according to quartiles and 1-SD increment of sFas levels.

Results: During the follow-up (1988 to 1997), we identified 233 (121 in men and 112 in women) deaths from total stroke, comprising of 49 (18 and 31) subarachnoid hemorrhages, 55 (27 and 28) intraparenchymal hemorrhages, and 71 (44 and 27) ischemic strokes, and 97 (53 and 44) coronary heart diseases. After adjustment for cardiovascular risk factors, the multivariable odds ratio $(95 \% \mathrm{CI})$ of subarachnoid hemorrhage associated with a $1-\mathrm{SD}$ increment of sFas $(1.3 \mathrm{ng} / \mathrm{ml}$ in both men and women) was $4.04(1.07-15.3 ; P=0.04)$. No association was found between blood sFas levels and risks of intraparenchymal hemorrhage, ischemic stroke or coronary heart disease. Conclusions: Higher blood sFas levels were associated with higher mortality from subarachnoid hemorrhage, suggesting a potential role of apoptosis factors in the development or prognosis of subarachnoid hemorrhage. 


\section{Introduction}

One of the major challenges in cardiovascular medicine is to develop a tool for predicting the risk that an individual will suffer an acute cardiovascular event. During the last few decades, there has been a great interest in finding diagnostic and prognostic biomarkers that can be detected in blood long before an event may occur (1-3). In addition to established biomarkers such as lipid and glucose abnormalities, new biomarkers have been explored to enhance prediction for cardiovascular disease. The Fas (also called Apo-1 or CD95) system is an apoptosis-signaling receptor located on the cell surface that belongs to the tumor necrosis factor receptor family (4). Apoptosis may be linked to the pathogenesis of cardiovascular disease (CVD) (5) including subarachnoid hemorrhage (6,7); however no evidence has been available on the prospective associations between blood sFas and risk of CVD. Most of the previous studies were limited to

describe an activation of the Fas system after the onset of coronary heart disease (CHD) (8-10), congestive heart failure $(11,12)$ and cerebrovascular diseases $(13,14)$.

We therefore investigated the associations between blood sFas levels and mortality from stroke and its subtypes including subarachnoid hemorrhage among middle-aged Japanese men and women by a nested-case control study under a nation-wide large prospective study.

\section{Methods}

\section{Survey Population}

We conducted a nested case-control study as part of the Japan Collaborative Cohort Study (JACC) sponsored by the Ministry of Education and Science, which was first conducted in 19881990 and involved 110,585 participants (46,395 men and 64,190 women, age 40-79 years) living in 45 communities throughout Japan who completed self-administered questionnaires about their lifestyles and medical histories of previous cardiovascular disease and cancer (15). Informed 
consent was obtained from the participants when they completed the questionnaire. In several communities, informed consent was obtained at community level after the study purpose, methods and data confidentiality had been explained to community leaders and mayors.

A total of 39,242 subjects (35.4\% of the questionnaire respondents) provided blood samples after giving individual informed consent (16). After 557 men and 916 women with a history of cardiovascular disease or cancer at baseline had been excluded, a total of 37,769 subjects (13,282 men and 24,487 women) were enrolled in the study presented here, which was approved by the Ethics Committees of the University of Tsukuba and Osaka University.

\section{Mortality Surveillance}

The participants were followed up to determine mortality of cardiovascular disease by the end of 1997. For mortality surveillance in individual communities, investigators conducted systematic reviews of the death certificates, all of which were forwarded to the public health center in the area of residency. Mortality data were centralized at the Ministry of Health and Welfare, and the underlying cause of death was coded according to the International Classification of Diseases (ICD), 9th revision, for deaths from 1988 to 1994, and the 10th revision for deaths from 1995 to 1997 as used for the National Vital Statistics. Registration of death is required by the Family Registration Law of Japan and is believed to be adhered to throughout Japan. All deaths occurring in the cohort were thus ascertained by death certificates from a public health center, except for subjects who died after moving away from their original community and were treated as censored cases.

The ICD 9th and 10th revisions were used to determine cause-specific mortality of cardiovascular disease as follows: coronary heart disease (410 to 414, I20 to I25) and total stroke (ICD 9th revision, codes 430 to 438, ICD 10th revision, codes I60 to I69) including 
intraparenchymal hemorrhage (431, I61), subarachnoid hemorrhage (430, I60), ischemic stroke (433 to 434, I63) and unclassified type of stroke. For each case, one control subject was selected randomly from participants without mortality from stroke or coronary heart disease, and matched for sex, age ( $\pm 5 y$ years), community and year of blood drawing.

\section{Determination of biochemical variables}

Sera were prepared from blood samples as soon as possible after blood collection at laboratories in or near the surveyed municipalities. The serum from each participant was divided over 3-5 tubes (300 $\mu \mathrm{l}$ per tube), and stored at $-80^{\circ} \mathrm{C}$ until analysis in a single laboratory (SRL, Inc., Hachioji, Japan); none of the samples had been defrosted (17). Blood sFas levels were measured by enzyme-linked immuno-adsorbent assay (ELISA), using commercially available kits (MBL Co., Ltd., Nagoya). Since sFas levels were systematically low in one area (no. of stroke cases =2), we excluded all sera from that area from the analysis. All assays were performed and interpreted by individuals who were blinded to the case-control status of samples (18). The intra-assay precisions obtained using different reference sera for each determination method was $2.1 \%$ to $5.5 \%$ of the coefficients variation values. The inter-assay coefficient of variance was $8.2 \%$ to $12.3 \%$.

Serum total cholesterol was measured using the enzymatic method with an automatic analyzer (Hitachi 7600-210; Hitachi Medical Corp., Tokyo, Japan) at Kotobiken Medical Laboratories, Inc. The standardization of lipid measurement was performed with the aid of the Osaka Medical Center for Health Science and Promotion, an international member of the US National Cholesterol Reference Method Laboratory Network (CRMLN) (19).

\section{Statistical Analysis}


The paired Student's t-test was used to compare the mean values of baseline cardiovascular risk characteristics and blood sFas levels in cases and control subjects. The $\chi^{2}$ test was used to compare percentages of cases and control subjects. Also in control subjects, differences in baseline risk characteristics were tested according to the sex-specific quartiles of blood sFas levels by the analysis of covariance. The odds ratios (ORs) and corresponding 95\% confidence intervals (CIs) for mortality from total stroke, stroke subtype and coronary heart disease according to the sex-specific quartiles and 1-SD increment of sFas were calculated by means of conditional logistic regression models. Cut points of the quartile analyses were determined on the distribution of control subjects. Linear regression was employed to test for linear trends across sFas categories by using a median variable of sFas for each biomarker category. Covariates for the adjustment included serum total cholesterol levels (mmol/L: continuous), cigarette smoking status (non and current), current ethanol intake (non and current), and self-reported histories of physician’s diagnosis hypertension and diabetes mellitus (yes vs. no) as well as matching for sex, age, area of residence and year of serum storage.

All p-values for statistical tests were two-tailed and values $<0.05$ were considered to represent represented statistical significance. The SAS statistical package version 9.4 (Statistical Analysis System Inc., Cary, NC) was used for analyses.

\section{Results}

During the median 9-year follow-up, we identified 97 (53 and 44) deaths from coronary heart diseases and 233 deaths from total stroke (121 in men and 112 in women) including 49 (18 and 31) subarachnoid hemorrhages, 55 (27 and 28) intraparenchymal hemorrhages, and 71 (44 and 27) ischemic strokes. 
Table 1 shows mean values and prevalence of cardiovascular risk characteristics and biomarkers for cases compared with control subjects. The average age was 68 years for total stroke, 62 years for subarachnoid hemorrhage, 67 years for intraparenchymal hemorrhage, 70 years for ischemic stroke, and 67 for coronary heart disease. The prevalence of hypertension and current smokers was higher in cases with total stroke than controls; the case-control difference in hypertension was evident for hemorrhagic strokes, and that in smoking was so for ischemic stroke. The prevalence of diabetes mellitus tended to be higher in cases with coronary heart disease than controls. Mean serum total cholesterol levels were lower in cases with total stroke, especially intraparenchymal hemorrhage than in controls. Mean sFas levels were higher in cases with total stroke, especially subarachnoid hemorrhage than controls. Risk characteristics according to sex-specific quartiles of blood sFas levels in the control subjects are shown in

\section{Supplemental Table 1.}

Table 2 presents age-, sex- and community-matched, as well as multivariable odds ratios (95\% CIs) for mortality from total stroke, stroke types and coronary heart disease by quartiles of and 1SD increment in sFas levels. Blood sFas levels were inversely associated with mortality from subarachnoid hemorrhage in both quartile and continuous analyses. Even after further adjustment for cardiovascular risk factors, the association with sFas remained statistically significant. The multivariable odds ratio (95\%CI) for subarachnoid hemorrhage for the highest vs. lowest quartiles of sFas was $3.07(0.52-18.0$; P for trend $=0.07)$, and that for $1-\mathrm{SD}$ increment $(1.3 \mathrm{ng} / \mathrm{ml}$ in both men and women) of sFas was $4.04(1.07-15.3 ; \mathrm{P}=0.04)$.

The association between sFas levels and mortality from subarachnoid hemorrhage was similarly observed for men and women with no sex interaction; $\mathrm{P}$ for interaction $=0.52$ (not shown in table). There were no associations of sFas levels with mortality from intraparenchymal hemorrhage, ischemic stroke or coronary heart disease. 


\section{Discussion}

Our nested case-control study performed as part of a large prospective study showed a positive association between Blood levels of sFas and risk of mortality from subarachnoid hemorrhage. These associations persisted even after major cardiovascular risk factors were taken into account, and similarly observed for both men and women.

The scope of previous studies that examined the association between sFas and CVD was limited to describe an activation of the Fas system after the onset of cardiovascular events that fades gradually after the case is stabilized (8-12). None of those studies has examined the prospective associations between sFas and cardiovascular endpoints. To our knowledge, the current nested case-control study is the first to show a prospective positive association between blood sFas and risk of subarachnoid haemorrhage.

Mechanisms for the increased risk of mortality from subarachnoid hemorrhage associated with higher blood sFas levels are suggested as follows. The expressions of Fas-associated death domain protein, the cytoplasmic domain of Fas, as well as tumor necrosis factor $\alpha$ were increased in cerebral aneurysm samples of patients with subarachnoid hemorrhage compared with samples of superficial temporal arteries of control subjects $(20,21)$. Since apoptosis is a key pathology for cerebral aneurysm in rats (22) and humans (23), in particular ruptured aneurysms (24), our finding on the associations of blood sFas levels with risk of subarachnoid hemorrhage is plausible. Blood sFas could be a promising biomarker for detecting subjects at high risk of subarachnoid hemorrhage.

Blood sFas levels, however, were not associated with risk of other types of stroke, or risk of coronary heart disease, while they were shown to be increased in subjects with or at high risk of ischemic heart disease (8-10). Moreover, one study showed that sFas levels were associated with 
risk of ischemic stroke; HR (95\% CI) for 1- SD increment was 1.31 (1.13-1.51; P=0.004) (25). Further research is needed to configure the mechanisms by which blood sFas associates with ischemic and hemorrhagic cardiovascular events.

The strengths of the present study are its prospective design for large community-based samples, the comprehensive nature of the cardiovascular surveys for exposure and confounding variables, the systematic surveillance for mortality outcomes and measuring the biomarkers of interest in a single laboratory with satisfactory reliability and precision.

However, our study has several limitations. First, approximately only 35\% of the total participants in health examinations with high participant rates had provided blood samples. However, no apparent differences were found in age-adjusted mean values or proportions of major cardiovascular risk factors between the subjects who had and who had not provided blood samples (16). Thus, the selection bias may be small for the evaluation of the biomarkerscardiovascular mortality association. Second, we used frozen serum to estimate biomarkers concentrations and did not examine long-term changes in the stored serum samples. We also confirmed that those serum biomarkers remained stable in serum samples stored up to 9 years at $70^{\circ} \mathrm{C}$ (17). Third, the association of borderline statistical significance with sFas levels in the multivariable categorical analysis could be attributable to the low statistical power. The analysis for 1-SD increment of log-transformed blood levels of sFas showed significant inverse associations. Last, we used the mortality data, not incidence data as endpoints, which biased to severe types of stroke and also liable to misclassification. As for stroke, however, the widespread use of computed tomography scans, even in Japanese local hospitals, since the 1980s has probably made the death certificate diagnosis of total stroke and stroke types sufficiently accurate (26). Death certificate diagnosis of coronary heart disease may be more problematic since onefourth to one-third of the coronary heart disease deaths appearing on the death certificates were 
contaminated $(27,28)$. The lack of association between biochemical markers and mortality from coronary heart disease might be in part due to the contamination of diagnosis and also the small number of cases.

In summary, Blood sFas levels were associated with higher mortality from subarachnoid hemorrhage among middle-aged men and women. Our findings support the notion that apoptosis may contribute in the development or prognosis of subarachnoid hemorrhage. 
Conflict of Interest: None declared for all authors.

\section{Financial support:}

This study was supported by Grants-in-Aid for Scientific Research from the Ministry of Education, Science, Sports and Culture of Japan; Grants-in-Aid for Scientific Research on Priority Areas of Cancer and Grants-in-Aid for Scientific Research on Priority Areas of Cancer Epidemiology from the Japanese Ministry of Education, Culture, Sports, Science and Technology (61010076, 62010074, 63010074, 1010068, 2151065, 3151064, 4151063, 5151069, 6279102, 11181101, 17015022, 18014011, 20014026, and 20390156); and Comprehensive Research on Cardiovascular and LifeStyle Related Diseases (H26-Junkankitou [Seisaku]-Ippan-001).

\section{Author's contribution}

Hiroyasu Iso and Akiko Tamakoshi participated in the study concept and design and acquisition of data. Hiroyasu Iso, Maruyama Koutatsu and Ehab S. Eshak participated in the study analysis and interpretation of data and drafting of the manuscript, and provided statistical expertise.

Satoyo Ikehara and Kazumasa Yamagioshi participated in interpretation of data, and critical revision of the manuscript.

\section{Acknowledgments}

The authors thank Dr. Yoshinori Ito, of Nagoya University Graduate School of Medicine, Dr. Yoshiyuki Watanabe, of Kyoto Prefectural University of Medicine Graduate School of Medical Science, Dr. Yutaka Inaba, of Jissen Women’s University, Dr. Kazuo Tajima, of Aichi Cancer Center Research Institute, Dr. Kei Nakachi, of Radiation Effects Research Foundation, and all staff members involved in this study for their valuable help in conducting the baseline survey and follow-up.

\section{Study Investigators}


Members of the JACC Study Group: Dr. Akiko Tamakoshi (present chairperson of the study group), Hokkaido University Graduate School of Medicine, Dr. Mitsuru Mori, Sapporo Medical University School of Medicine, Dr. Yoshihiro Kaneko, Akita University Graduate School of Medicine, Dr. Ichiro Tsuji, Tohoku University Graduate School of Medicine, Dr. Yosikazu Nakamura, Jichi Medical School, Dr. Hiroyasu Iso, Osaka University School of Medicine, Dr, Kazumasa Yamagishi, Faculty of Medicine, University of Tsukuba, Dr. Haruo Mikami, Chiba Cancer Center, Dr. Michiko Kurosawa, Juntendo University School of Medicine Dr. Yoshiharu Hoshiyama, Yokohama Soei University, Dr. Naohito Tanabe, University of Niigata Prefecture, Dr. Koji Tamakoshi, Nagoya University Graduate School of Health Science, Dr. Kenji Wakai, Nagoya University Graduate School of Medicine, Dr. Shinkan Tokudome, National Institute of Health and Nutrition, Dr. Koji Suzuki, Fujita Health University School of Health Sciences, Drs. Shuji Hashimoto and Hiroshi Yatsuya, Fujita Health University School of Medicine, Dr. Shogo Kikuchi, Aichi Medical University School of Medicine, Dr. Yasuhiko Wada, Faculty of Nutrition, University of Kochi, Dr. Takashi Kawamura, Kyoto University Health Service, Dr. Yoshiyuki Watanabe, Kyoto Prefectural University of Medicine Graduate School of Medical Science, Dr. Kotaro Ozasa, Radiation Effects Research Foundation, Dr. Kazuya Mikami, Kyoto Prefectural University of Medicine Graduate School of Medical Science, Dr. Chigusa Date, School of Human Science and Environment, University of Hyogo, Dr. Kiyomi Sakata, Iwate Medical University, Dr. Yoichi Kurozawa, Tottori University Faculty of Medicine, Drs. Takesumi Yoshimura and Yoshihisa Fujino, University of Occupational and Environmental Health, Dr. Akira Shibata, Kurume University, Dr. Naoyuki Okamoto, Kanagawa Cancer Center, and Dr. Hideo Shio, Long-Term Care Health Facility Caretown Minamikusatsu, Shiga. 


\section{REFRENCES}

1- van der Meer IM, Oei H-HS, Hofman A, Pold HAP, de Jog FH, et al. Soluble Fas, a mediator of apoptosis, C-reactive protein, and coronary and extracoronary atherosclerosis. The Rotterdam Coronary Calcification Study. Atherosclerosis 2006;189:464-469.

2-Martín-Ventura JL, Blanco-Colio LM, Tuñón J, Muñoz-García B, Madrigal-Matute J, et al. Biomarkers in cardiovascular medicine. Rev Esp Cardiol. 2009;62:677-688.

3- Iso H, Cui R, Date C, Kikuchi S, Tamakoshi A. C-reactive protein levels and risk of mortality from cardiovascular disease in Japanese: the JACC Study. Atherosclerosis. 2009;207:291-297.

4- Nagata S, Golstein P. The Fas death factor. Science. 1995;267:1449-1456.

5- Lee Y, Gustafsson AB. Role of apoptosis in cardiovascular disease. Apoptosis. 2009;14:536548.

6- Sabri M, Kawashima A, Ai J, Macdonald RL. Neuronal and astrocytic apoptosis after subarachnoid hemorrhage: a possible cause for poor prognosis. Brain Res. 2008;1238:163-1671.

7- Hasegawa Y, Suzuki H, Sozen T, Altay O, Zhang JH. Apoptotic mechanisms for neuronal cells in early brain injury after subarachnoid hemorrhage. Acta Neurochir Suppl. 2011;110(Pt 1):43-48.

8-Shimizu M, Fukuo K, Nagata S, Suhara T, Okuro M, et al. Increased plasma levels of the soluble form of Fas ligand in patients with acute myocardial infarction and unstable angina pectoris. J Am Coll Cardiol. 2002;39:585-590.

9- Hébert MJ, Masse M, Vigneault N, Sirois I, Troyanov S, et al. Soluble Fas is a marker of coronary artery disease in patients with end-stage renal disease. Am J Kidney Dis. 2001;38:12711276.

10- Sahinarslan A, Boyaci B, Kocaman SA, Topal S, Ercin U, et al. The relationship of serum soluble Fas ligand (sFasl) level with the extent of coronary artery disease. Int J Angiol. 2012;21:29-34.

11- Nishigaki K, Minatoguchi S, Seishima M, Asano K, Noda T, et al. Plasma Fas ligand, an inducer of apoptosis, and plasma soluble Fas, an inhibitor of apoptosis, in patients with chronic congestive heart failure. J Am Coll Cardiol. 1997;29:1214-1220.

12- Niessner A, Hohensinner PJ, Rychli K, Neuhold S, Zorn G, et al. Prognostic value of apoptosis markers in advanced heart failure. Eur Heart J. 2009;30:789-796.

13- Delgado P, Cuadrado E, Rosell A, Alvarez-Sabín J, Ortega-Aznar A, et al. Fas system activation in perihematomal areas after spontaneous intracerebral hemorrhage. Stroke. 2008;39:1730-1734. 
14- Mahovic D, Zurak N, Lakusic N, Sporis D, Zarkovic N, et al. The dynamics of soluble Fas/APO 1 apoptotic biochemical marker in acute ischemic stroke patients. Adv Med Sci. 2013;58:298-303.

15- Ohno Y, Tamakoshi A and the JACC Study Group. Japan collaborative cohort study for evaluation of cancer risk sponsored by Monbusho (JACC study). J Epidemiol 2001; 11:144-150.

16- Iso H, Cui R, Date C, Kikuchi S, Tamakoshi A. C-reactive protein levels and risk of mortality from cardiovascular disease in Japanese: the JACC Study. Atherosclerosis. 2009 ;207:291-297.

17- Ito Y, Nakachi K, Imai K, Hashimoto S, Watanabe Y, et al. Satability of frozen serum levels of insulin-like growth factor-1, insulin-like growth factor-II, insulin-like growth factor binding protein-3, transforming growth factor beta, soluble Fas, and superoxide dismutase activity for the JACC study. J Epidemiol 2005;15(Suppl 1):S67-73.

18- Tamakoshi A, Nakachi K, Ito Y, Lin Y, Yagyu K, et al. Soluble Fas level and cancer mortality: findings from a nested case-control study within a large-scale prospective study. Int J Cancer. 2008 15;123:1913-1916.

19- Nakamura M, Sato S, Shimamoto T. Improvement in Japanese clinical laboratory measurements of total cholesterol and HDL-cholesterol by the US Cholesterol Reference Method Laboratory Network. J Atheroscler Thromb. 2003;10:145-153.

20- Jayaraman T, Berenstein V, Li X, Mayer J, Silane M, et al. Tumor necrosis factor alpha is a key modulator of inflammation in cerebral aneurysms. Neurosurgery. 2005;57:558-64.

21-Starke RM, Raper DM, Ding D, Chalouhi N, Owens GK, et al. Tumor necrosis factor- $\alpha$ modulates cerebral aneurysm formation and rupture. Transl Stroke Res. 2014;5:269-277.

22- Kondo S, Hashimoto N, Kikuchi H, Hazama F, Nagata I, et al. Apoptosis of medial smooth muscle cells in the development of saccular cerebral aneurysms in rats. Stroke 1998;29:181-189.

23- Hara A, Yoshimi N, Mori H. Evidence for apoptosis in human intracranial aneurysms. Neurol Res 1998;20:127-130.

24- Pentimalli L, Modesti A, Vignati A, Marchese E, Albanese A, et al. Role of apoptosis in intra cranial aneurysm rupture. J Neurosurg. 2004;101:1018-1025.

25-Lind L, Siegbahn A, Lindahl B, Stenemo M, Sundström J, et al. Discovery of new risk markers for ischemic stroke using a novel targeted proteomics chip. Stroke. 2015;46:3340-3347.

26- Sasamamoto T, Iida M, Tanigaki M, Naito Y, et al. A population-based study of the proportion by type of stroke determined by computed tomography scan. Nippon Koshu Eisei Zasshi 1991;38:901-909. 
27- Yamashita T, Ozawa H, Aono H, Hosokawa H, Saito I, et al. Heart disease deaths on death certificates re-evaluated by clinical records in a Japanese city. CHD diagnosis. Jpn Circ J 1997;61:331-338.

28-Baba S, Ozawa H, Sakai Y, Terao A, Konishi M, et al. Heart disease deaths in a Japanese urban area evaluated by clinical and police records. Circulation 1994; 89:109-115. 
Table 1. Mean values \pm standard deviations and proportions of cardiovascular risk characteristics and blood sFas levels in cases and controls.

\begin{tabular}{|c|c|c|c|c|c|c|c|c|c|c|}
\hline & \multicolumn{2}{|c|}{ Total stroke } & \multicolumn{2}{|c|}{ Subarachnoid hemorrhage } & \multicolumn{2}{|c|}{ Intraparenchymal hemorrhage } & \multicolumn{2}{|c|}{ Ischemic stroke } & \multicolumn{2}{|c|}{ Coronary heart disease } \\
\hline & Cases & Controls & Cases & Controls & Cases & Controls & Cases & Controls & Cases & Controls \\
\hline No. of subjects & 233 & 233 & 49 & 49 & 55 & 55 & 71 & 71 & 97 & 97 \\
\hline Men, \% & 51.9 & 51.9 & 36.7 & 36.7 & 50.9 & 50.9 & 62.0 & 62.0 & 54.6 & 54.6 \\
\hline Age, year & $67.7 \pm 8.1$ & $67.0 \pm 7.5$ & $61.9 \pm 9.7$ & $61.8 \pm 9.4$ & $66.6 \pm 7.8$ & $66.3 \pm 7.5$ & $70.3 \pm 6.4$ & $69.1 \pm 5.5$ & $67.3 \pm 7.6$ & $66.9 \pm 7.3$ \\
\hline Total cholesterol, mmol/L & $4.98 \pm 1.01^{\mathrm{b}}$ & $5.26 \pm 1.03$ & $5.01 \pm 0.94$ & $5.32 \pm 1.25$ & $4.83 \pm 1.12^{\mathrm{a}}$ & $5.31 \pm 1.00$ & $5.01 \pm 0.91$ & $5.19 \pm 0.90$ & $5.42 \pm 0.99$ & $5.26 \pm 1.17$ \\
\hline Current smoker, \% & $38.4^{\mathrm{a}}$ & 27.0 & 31.9 & 23.9 & 34.0 & 27.1 & $47.6^{\mathrm{a}}$ & 27.3 & 41.4 & 33.7 \\
\hline Ethanol intake, g/day & $35 \pm 25$ & $31 \pm 23$ & $33 \pm 21$ & $28 \pm 25$ & $41 \pm 34$ & $28 \pm 22$ & $29 \pm 19$ & $31 \pm 19$ & $31 \pm 23$ & $26 \pm 24$ \\
\hline History of hypertension, \% & $44.9^{\mathrm{b}}$ & 31.5 & $44.4^{\mathrm{a}}$ & 23.4 & $44.0^{\mathrm{a}}$ & 24.5 & 45.3 & 32.8 & 41.4 & 33.7 \\
\hline History of diabetes mellitus, \% & 5.3 & 5.7 & 9.3 & 4.4 & 0.0 & 2.1 & 3.3 & 9.5 & 12.8 & 4.6 \\
\hline sFas, ng/ml & $2.4 \pm 1.4$ & $2.2 \pm 1.3$ & $2.3 \pm 1.4^{\mathrm{a}}$ & $2.0 \pm 1.3$ & $2.6 \pm 1.4^{\mathrm{a}}$ & $2.4 \pm 1.3$ & $2.4 \pm 1.4$ & $2.3 \pm 1.4$ & $2.2 \pm 1.4$ & $2.2 \pm 1.3$ \\
\hline
\end{tabular}

Difference from controls: ${ }^{\mathrm{a}} \mathrm{p}<0.05,{ }^{\mathrm{b}} \mathrm{p}<0.01$. 
Table 2. Conditional odds ratios (ORs) and $95 \%$ confidence intervals (CIs) for mortality from

5 total stroke, stroke types and coronary heart disease according to quartiles of sFas levels.

Quartiles of blood soluble Fas levels ${ }^{\text {a }}$

OR per 1 SD $^{\mathrm{b}}$

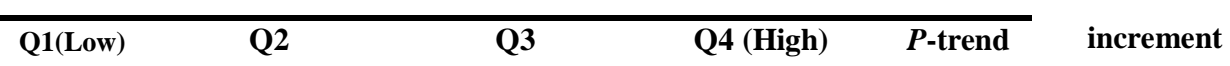

\begin{tabular}{|c|c|c|c|c|c|c|c|}
\hline \multicolumn{8}{|l|}{ Total stroke } \\
\hline No. of cases & 51 & 54 & 52 & 76 & & & \\
\hline No. of controls & 54 & 66 & 57 & 56 & & & \\
\hline Age, sex and community-matched OR & 1.00 & $0.91(0.53-1.57)$ & $1.01(0.56-1.81)$ & $1.55(0.87-2.75)$ & 0.07 & $1.51(1.06-2.16)$ & 0.02 \\
\hline Multivariable $\mathrm{OR}^{\mathrm{c}}$ & 1.00 & $0.96(0.53-1.74)$ & $1.13(0.59-2.16)$ & $1.38(0.74-2.58)$ & 0.22 & $1.37(0.92-2.03)$ & 0.12 \\
\hline \multicolumn{8}{|l|}{ Subarachnoid hemorrhage } \\
\hline No. of cases & 13 & 11 & 10 & 15 & & & \\
\hline No. of controls & 18 & 16 & 6 & 9 & & & \\
\hline Age, sex and community-matched OR & 1.00 & $1.10(0.35-3.48)$ & $2.90(0.70-12.0)$ & $3.40(0.82-14.1)$ & 0.05 & $3.40(1.12-10.3)$ & 0.03 \\
\hline Multivariable OR ${ }^{\mathrm{c}}$ & 1.00 & $1.21(0.21-6.86)$ & $4.44(0.72-27.4)$ & $3.07(0.52-18.0)$ & 0.07 & $4.04(1.07-15.3)$ & 0.04 \\
\hline \multicolumn{8}{|l|}{ Intraparenchymal hemorrhage } \\
\hline No. of cases & 6 & 14 & 13 & 22 & & & \\
\hline No. of controls & 6 & 15 & 20 & 14 & & & \\
\hline Age, sex and community-matched OR & 1.00 & $1.23(0.26-5.76)$ & $0.74(0.16-3.29)$ & $1.84(0.38-8.96)$ & 0.27 & $1.53(0.82-2.85)$ & 0.18 \\
\hline Multivariable OR ${ }^{\mathrm{c}}$ & 1.00 & $1.72(0.20-14.7)$ & $0.65(0.67-6.43)$ & $1.23(0.13-11.5)$ & 0.95 & $0.86(0.34-2.17)$ & 0.75 \\
\hline \multicolumn{8}{|l|}{ Ischemic stroke } \\
\hline No. of cases & 20 & 13 & 13 & 25 & & & \\
\hline No. of controls & 14 & 22 & 15 & 20 & & & \\
\hline Age, sex and community-matched OR & 1.00 & $0.37(0.13-1.06)$ & $0.54(0.19-1.55)$ & $0.93(0.36-2.42)$ & 0.81 & $1.29(0.72-2.30)$ & 0.39 \\
\hline Multivariable $\mathrm{OR}^{\mathrm{c}}$ & 1.00 & $0.49(0.14-1.65)$ & $0.68(0.2-2.31)$ & $1.26(0.41-3.89)$ & 0.45 & $1.69(0.85-3.36)$ & 0.13 \\
\hline \multicolumn{8}{|l|}{ Coronary heart disease } \\
\hline No. of cases & 30 & 18 & 22 & 27 & & & \\
\hline No. of controls & 24 & 28 & 22 & 23 & & & \\
\hline Age, sex and community-matched OR & 1.00 & $0.55(0.25-1.21)$ & $0.78(0.35-1.73)$ & $1.02(0.40-2.63)$ & 0.84 & $1.00(0.56-1.78)$ & 1.00 \\
\hline Multivariable $\mathrm{OR}^{\mathrm{c}}$ & 1.00 & $0.46(0.19-1.10)$ & $0.73(0.28-1.89)$ & $0.78(0.25-2.42)$ & 0.86 & $0.81(0.40-1.66)$ & 0.56 \\
\hline
\end{tabular}

${ }^{\mathrm{a}}$ Ranges of $\mathrm{sFas}$ in quartiles were $\leq 1.9,1.9-2.3,2.3-2.9,2.9+\mathrm{ng} / \mathrm{ml}$ in men and $\leq 1.9,1.9-2.3,2.3-2.7,2.7+\mathrm{ng} / \mathrm{ml}$ in women.

${ }^{\mathrm{b}} 1-\mathrm{SD}$ of of sFas were $1.3 \mathrm{ng} / \mathrm{ml}$ in men and $1.3 \mathrm{ng} / \mathrm{ml}$ in women.

c Multivariable OR: further adjusted for current smoking status (yes/no), alcohol consumption (current/non-drinker), serum total

10 cholesterol levels (continuous) and history of diabetes mellitus and hypertension (yes/no). 
Supplemental Table 1. Age- and sex-adjusted mean values (standard errors) and proportions of cardiovascular risk characteristics in control subjects according to quartiles of blood soluble Fas levels.

\begin{tabular}{|c|c|c|c|c|c|}
\hline & \multicolumn{5}{|c|}{ Quartiles of blood soluble Fas levels ${ }^{a}$} \\
\hline & Q1(Low) & Q2 & Q3 & Q4 (High) & $P$-trend \\
\hline No. of subjects & 78 & 94 & 79 & 79 & \\
\hline Men, \% & 51.3 & 52.1 & 54.4 & 53.2 & 0.98 \\
\hline Age, year & 66.1 & 65.6 & 67.1 & 69.4 & $<0.01$ \\
\hline Total cholesterol, mmol/L & $5.2(0.12)$ & $5.3(0.11)$ & $5.3(0.12)$ & $5.2(0.12)$ & 0.82 \\
\hline Current smoker, \% & 23.6 & 29.6 & 22.2 & 33.9 & 0.23 \\
\hline Ethanol intake, g/day & $22.0(3.93)$ & $22.5(4.05)$ & $20.9(4.74)$ & $18.3(6.45)$ & 0.60 \\
\hline Body mass index, $\mathrm{kg} / \mathrm{m}^{2}$ & $22.3(0.36)$ & $22.7(0.33)$ & $23.0(0.37)$ & $22.9(0.37)$ & 0.20 \\
\hline History of hypertension, \% & 24.3 & 31.6 & 35.3 & 38.8 & 0.06 \\
\hline History of diabetes mellitus, \% & 8.1 & 4.9 & 2.9 & 5.4 & 0.44 \\
\hline sFas, ng/ml* & 1.5 & 2.0 & 2.5 & 3.2 & $<0.01$ \\
\hline
\end{tabular}

*Ranges of blood sFas in quartiles were $\leq 1.9,1.9-2.3,2.3-2.9,2.9+\mathrm{ng} / \mathrm{ml}$ in men and $\leq 1.9,1.9-2.3,2.3-2.7,2.7+\mathrm{ng} / \mathrm{ml}$ in women. 